\title{
The expression profile of Dopamine D2 receptor, MGMT and VEGF in different histological subtypes of pituitary adenomas: a study of 197 cases and indications for the medical therapy
}

Youwei Wang ${ }^{1,3+}$, Junyang $\mathrm{Li}^{1 \dagger}$, Mamatemin Tohti ${ }^{1}$, Yuebing $\mathrm{Hu}^{1}$, Sheng Wang ${ }^{1}$, Wanchun $\mathrm{Li}^{2}$, Zhenfeng $\mathrm{Lu}^{2}$ and Chiyuan $\mathrm{Ma}^{1 *}$

\begin{abstract}
Background: To study the expression of D2R, MGMT and VEGF for clinical significance in pituitary adenomas, and to predict the potential curative medical therapy of dopamine agonists, temozolomide and bevacizumab on pituitary adenomas.

Methods: Immunohistochemistry and western blot were performed to detect the expression of expression of D2R, MGMT and VEGF in pituitary adenoma tissue samples. The ratio of high expression of D2R, MGMT or VEGF in different subtypes of PA was compared by the use of chi-squared tests. The relationships between D2R, MGMT and VEGF expression were assessed by the Spearman rank correlation test. The association between their expression and clinical parameters was analyzed using a chi-squared test, or Fisher's exact probability test when appropriate.

Results: The data showed that in 197 different histological subtypes of pituitary adenomas (PAs), $64.9 \%$ of them were D2R high expression, 86.3\% were MGMT low expression and 58.9\% were VEGF high expression. D2R high expression existed more frequently in PRL- and GH- secreting PAs. MGMT low expression existed in all PA subtypes. VEGF high expression existed more frequently in PRL, ACTH, FSH secreting and non-functioning PAs. The data of western blot also support the results. Spearman's rank correlation analysis showed that expression of MGMT was positively associated with D2R $(r=0.154, P=0.031)$ and VEGF $(r=0.161, P=0.024)$ in PAs, but no correlation was showed between D2R and VEGF expression $(r=-0.025, P=0.725>0.05)$. The association between their expression and clinical parameters was analyzed using a chi-squared test, or Fisher's exact probability test when appropriate, but the result showed no significant association.

Conclusions: PRL-and GH-secreting PAs exist high expression of D2R, responding to dopamine agonists; Most PAs exist low expression of MGMT and high expression of VEGF, TMZ or bevacizumab treatment could be applied under the premise of indications.
\end{abstract}

Keywords: Dopamine D2 receptors, MGMT, VEGF, Dopamine agonists, Temozolomide, Bevacizumab

\footnotetext{
*Correspondence: machiyuan_nju@126.com

${ }^{\dagger}$ Equal contributors

'Department of Neurosurgery, Jinling Hospital, School of Medicine, Nanjing

University, 305 East Zhongshan Road, Nanjing 210002, China

Full list of author information is available at the end of the article
} 


\section{Background}

Pituitary adenomas (PAs) account for about 15\% of intracranial tumors. Although PAs are mostly benign lesions, about $30-55 \%$ of them are confirmed to locally invasive, and some of them infiltrate dura, bone and sinuses, are designated highly aggressive [1,2]. The conventional treatment of large pituitary adenomas consists of surgery, and radiotherapy when it is hard to achieve total resection. The use of additional radiotherapy is limited by the risk of radiation necrosis of surrounding structures. Thus, medication treatment, although unlikely to be curative immediately, might lead to certain clinically therapeutic effect, as a useful supplement [3].

Currently, first-line clinical medication for PAs generally consists of dopamine agonists (DAs), somatostatin analogs (SSAs) or combinations [4]. Recently, some routine chemotherapeutics such as Temozolomide (TMZ) and Bevacizumab have been carefully studied to treat PAs and considered to be potential for aggressive PAs' medical therapy [5-8]. DAs were widely used for the treatment of prolactinomas and some somatotropinomas, and the responsiveness depends on the expression of dopamine D2 receptors (D2R) on tumor cells. Abnormal expression of $\mathrm{D} 2 \mathrm{R}$ in prolactinoma was considered to confer resistance to DA treatment. Fadul et al. [7] first reported two cases of pituitary carcinoma received TMZ treatment, concluding that TMZ may be effective in treating pituitary carcinomas. After that, more and more studies demonstrated the inspiring therapeutic effect of TMZ on pituitary carcinomas and aggressive PAs. As a DNA repairase, O6-methylguanine DNA methyltransferase (MGMT) confers chemoresistance to TMZ [9]. Thus, tumors with low expression of MGMT are usually sensitive to TMZ. Bevacizumab is a monoclonal antibody which has been approved by USA FDA to treat colorectal cancer, non-small-cell lung carcinoma, breast cancer, renal carcinoma and recurrent glioma [10]. It blocks vascular endothelial growth factor (VEGF) binding to its receptor [11]. Experimental and clinical studies have demonstrated that anti-VEGF therapy may be effective in pituitary carcinoma and aggressive PAs.

To investigate D2R, MGMT and VEGF expression profile in PAs, and to evaluate the status of the drug targets of DAs, TMZ and Bevacizumab for PA medical therapy, herein, we performed the immunohistochemical staining in 197 cases of different subtypes of PAs.

\section{Methods}

\section{Patients and tissues}

One hundred and ninety seven pituitary adenomas (PAs) of different histological subtypes were selected randomly from patients operated between 2009 and 2011 in the Department of neurosurgery, Jinling Hospital, School of Medicine, Nanjing University. All PA tumor tissues were formalin-fixed and paraffinembedded resected and then pathologically diagnosed, including 28 PRL-secreting adenomas, $20 \mathrm{GH}$-secreting adenomas, $27 \mathrm{ACTH}$-secreting adenomas, 15 TSH-secreting adenomas, 37 FSH-secreting adenomas and 70 non-functioning adenomas.

\section{Immunohistochemical staining}

A streptavidin-peroxidase (SP) method was used for immunostaining. Briefly, slides were deparaffinized with xylene three times (each for 5-10 min), dehydrated three times in a gradient series of ethanol (100\%, 95\%, and 75\%), and rinsed with PBS. Each slide was treated with 3\% H2O2 for $15 \mathrm{~min}$ to quench endogenous peroxidase activity. Nonspecific bindings were blocked by treating slides with normal goat serum for $20 \mathrm{~min}$. Slides were first incubated with rabbit polyclonal anti-D2R (Abcam, Shanghai, China; 1:50), mouse monoclonal anti-MGMT (Abcam, Shanghai, China; 1:50) or mouse monoclonal anti-VEGF (Abcam, Shanghai, China; $1: 50$ ) overnight at $4^{\circ} \mathrm{C}$, and then rinsed twice with PBS. Slides were then incubated with a secondary antibody for $15 \mathrm{~min}$ at $37^{\circ} \mathrm{C}$ followed by treatment with streptavidin-peroxidase reagent for $15 \mathrm{~min}$, and rinsed twice with PBS. The slides were visualized with 3,3'-diaminobenzidine (DAB) for $3 \mathrm{~min}$, counterstained with haematoxylin, and mounted for microscopy.

\section{Evaluation of staining}

The slides were evaluated by two separate investigators under a light microscope (Dr. Wanchun Li and Dr. Zhenfeng Lu). Staining intensity was scored as 0 (negative), 1 (weak), 2 (medium), and 3 (strong). Extent of staining was scored as 0 (0\%), 1 (1-25\%), 2 (26-50\%), 3 (51-75\%), and 4 (76-100\%) according to the percentages of the positive staining areas in relation to the whole carcinoma area. The sum of the intensity score and extent score was used as the final staining score (0-7). Tumors having a final staining score of $>2$ were considered to be positive, score of 2-3 were considered as low expression and score of $>3$ were high expression.

\section{Western blot}

For western blot analysis, the lysates were separated by SDS-PAGE followed by transferring to an Immobilon-P Transfer membrane (Millipore Corporation, Bedford, MA, USA). Membranes were probed with primary antibodies followed by incubation with secondary antibody. Proteins were visualized with chemiluminescence luminol reagents (Beyotime Institute of Biotechnology, Shanghai, China).

\section{Statistical analysis}

Statistical analysis was performed using SPSS 16.0 (SPSS Chicago, IL, USA). The ratio of high expression of D2R, MGMT or VEGF in different subtypes of PA was compared by the use of chi-squared tests. The relationships 
between D2R, MGMT and VEGF expression were assessed by the Spearman rank correlation test. The association between their expression and clinical parameters was analyzed using a chi-squared test, or Fisher's exact probability test when appropriate. $\mathrm{P}<0.05$ was considered to be statistically significant.

\section{Results}

\section{Expression of D2R, MGMT or VEGF in PA tissues}

The location of D2R and VEGF in the nuclei and cytoplasm, and of MGMT in the nuclei was considered for scoring (Figure 1A-F). The positive expression of D2R was detected in 194 tissues, of MGMT was in all tissues and of VEGF was in 190 tissues. The proportions of cases showing low (score of $\leq 3$ ) or high (score of $>3$ ) expression levels for D2R, MGMT and VEGF in different subtypes of PA were shown in Table $1.64 .9 \%$ of 197 PAs were D2R high expression, $86.3 \%$ of them were MGMT low expression and $58.9 \%$ of them were VEGF high expression. The ratio of high expression of D2R or MGMT is significantly different in PA subtypes (For D2R: $X^{2}=44.844$, $\mathrm{P}<0.001$; For MGMT: $\left.\chi^{2}=13.210, \mathrm{P}=0.021\right)$, but for VEGF, there is no significance $\left(X^{2}=9.003, P=0.109\right)$. D2R high expression existed more frequently in PRL, GH, ACTH, TSH and FSH secreting PAs. MGMT low expression existed in all PA subtypes. VEGF high expression existed more frequently in PRL, ACTH, FSH secreting and non-functioning PA. The data of western blot supported and confirmed these results (Figure 2).

\section{Relationships between D2R, MGMT and VEGF expression in correlation analysis}

Spearman's rank correlation analysis showed that MGMT expression was positively associated with D2R expression $(r=0.154, P=0.031)$ and with VEGF expression $(\mathrm{r}=0.161, \mathrm{P}=0.024)$ in $\mathrm{PA}$, but $\mathrm{D} 2 \mathrm{R}$ expression did not show a correlation with VEGF expression $(r=-0.025$, $\mathrm{P}=0.725>0.05)$.

\section{Association of D2R, MGMT and VEGF expression with clinical features of PAs}

In these 197 cases, 106 of them were male and 91 were female; 64 of them were defined as invasive PAs, and others were non-invasive (according to Knosp's classification [12]); 16 of them were recurrent PA, and the others were primary; 16 of them were microadenoma
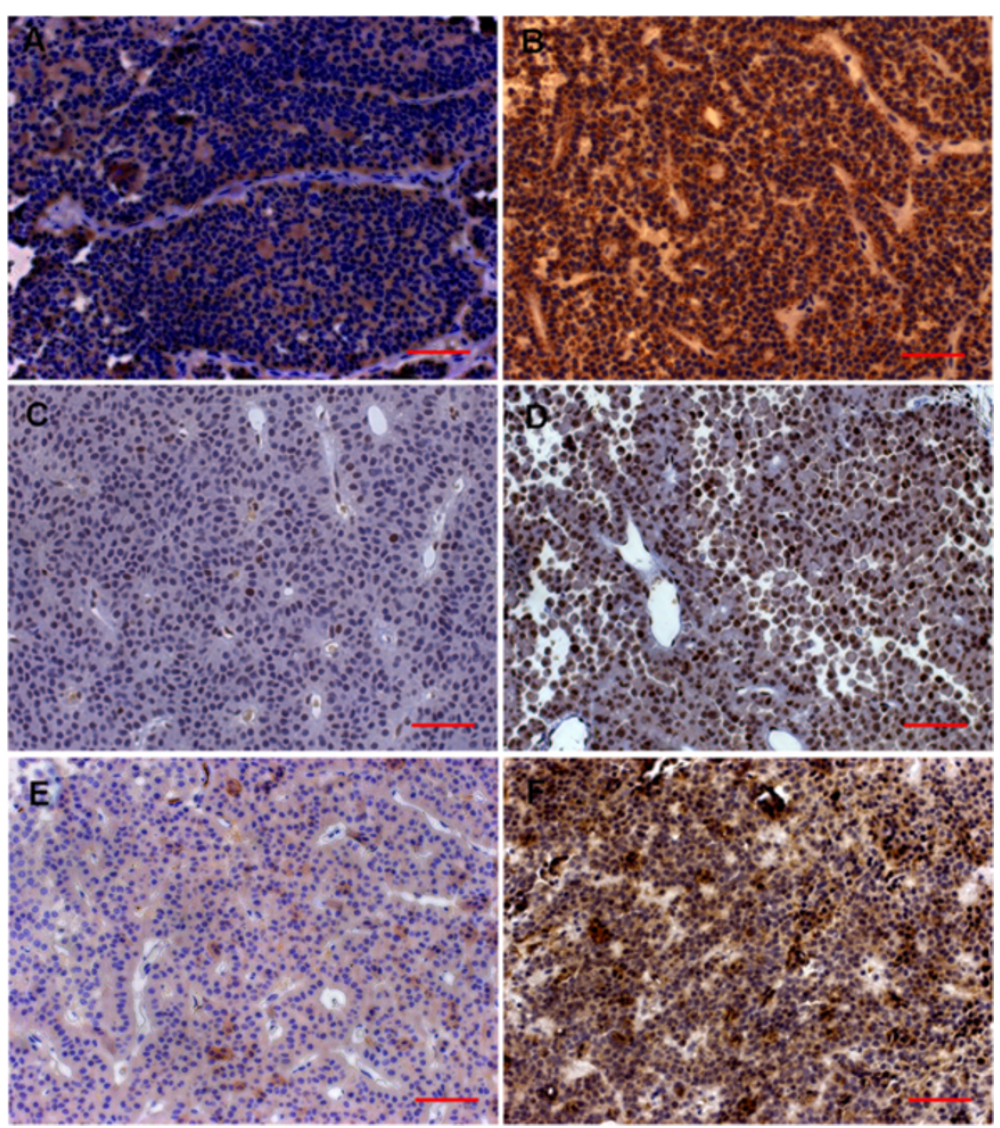

Figure 1 Expression of D2R, MGMT and VEGF in PAs. (A, B): D2R low (A) and high (B) expression. (C, D): MGMT low (C) and high (D) expression. (E, F): VEGF low (E) and high (F) expression. Bar $=50 \mu \mathrm{m}$. 
Table 1 Expression profile of D2R, MGMT and VEGF in different subtypes of PA

\begin{tabular}{|c|c|c|c|c|c|c|c|}
\hline \multirow[t]{2}{*}{ PA subtypes } & \multirow{2}{*}{$\begin{array}{l}\text { No. of } \\
\text { patients }\end{array}$} & \multicolumn{2}{|l|}{ D2R } & \multicolumn{2}{|c|}{ MGMT } & \multicolumn{2}{|c|}{ VEGF } \\
\hline & & Low & High & Low & High & Low & High \\
\hline PRL & 28 & 2 & 26 & 24 & 4 & 11 & 17 \\
\hline $\mathrm{GH}$ & 20 & 2 & 18 & 18 & 2 & 11 & 9 \\
\hline ACTH & 27 & 9 & 18 & 22 & 5 & 13 & 14 \\
\hline TSH & 15 & 6 & 9 & 14 & 1 & 8 & 7 \\
\hline FSH & 37 & 6 & 31 & 26 & 11 & 8 & 29 \\
\hline NF & 70 & 44 & 26 & 66 & 4 & 30 & 40 \\
\hline Total & 197 & 69 & 128 & 170 & 27 & 81 & 116 \\
\hline
\end{tabular}

$\mathrm{NF}$, Non-functioning; Low, low expression (score of $\leq 3$ ); High, high expression (score of $>3$ ).

(diameter $\leq 10 \mathrm{~mm}$ ), and the others were macroadenoma (diameter $>10 \mathrm{~mm}$ ); 159 of the PAs were tender in tumor tissues, and the others were tenacious; Only 8 patients have taken bromocriptine orally. The associations between clinical variables and D2R, MGMT and VEGF expression are shown in Table 2. However, there was no significant association between D2R, MGMT or VEGF expression and clinical features, including patient sex, tumor growth pattern, tumor recurrence, tumor size, tumor tissue texture and bromocriptine application $(\mathrm{P}>0.05)$. This indicated that despite the variety of PA clinical features, the expression of D2R, MGMT and VEGF are definite in PAs.

\section{Discussion}

Dopamine D2 receptor is expressed in the anterior and intermediate lobes of the pituitary gland. The response

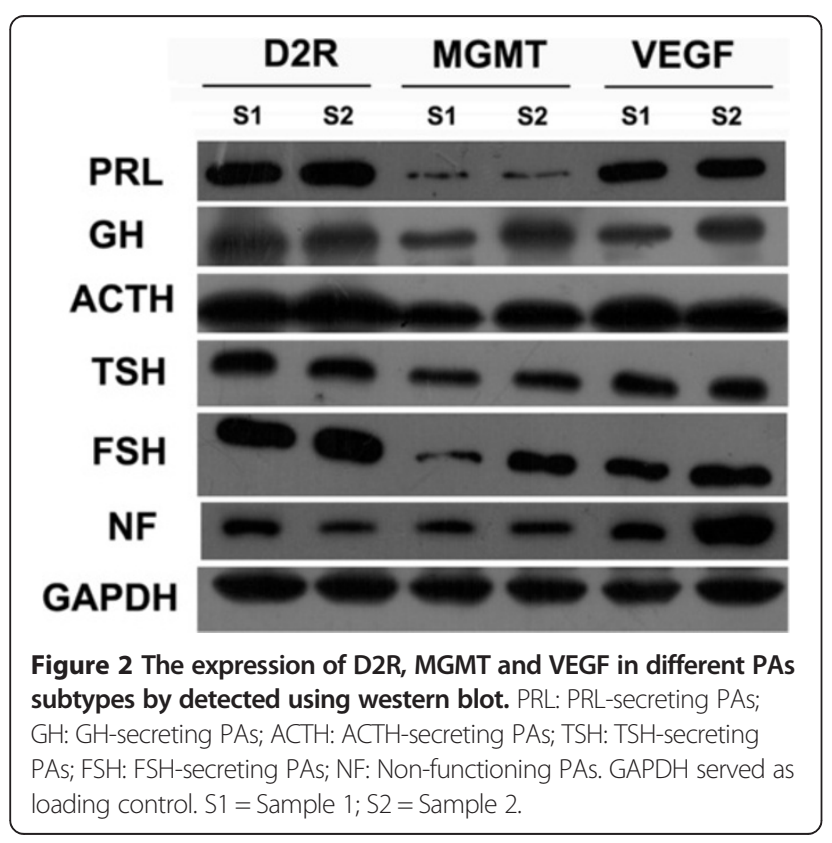

to dopamine agonists is related to the activity of the D2 receptor which belongs to the family of $G$ proteincoupled receptors and acts through AMP cyclase enzyme inhibition [13]. de Bruin et al. demonstrated that D2 receptor expressed in more than $75 \%$ of the cell population in normal human pituitary, indicating that D2 receptors are not expressed only in lactotrophs and melanotrophs, which represent no more than $30 \%$ of the entire cell population of the normal pituitary gland [14]. In PRL secreting pituitary tumors, the high espression level of D2 receptor explains the good therapeutic response to dopamine agonists, which induces tumor shrinkage. In present study, we investigated the expression of D2R in 197 cases of PAs and found that approximately 92.9\% of prolactinomas and $90 \%$ of somatotropinomas are high expression of D2R, indicating potential good drug-sensitivity for dopamine agonists (DAs). Previous clinical studies revealed that cabergoline and bromocriptine can normalize serum PRL levels in more than $80 \%$ of prolactinomas patients $[15,16]$ and have a good effect in somatotropinoma patients [17], which consistent with our data from immunostaining analysis. Our data also showed $83.8 \%$ of FSHsecreting PAs and $66.7 \%$ of ACTH-secreting PAs are high expression of D2R, which is supported by several other reported studies, although clinical studies showed a longterm cure of $48 \%$ in cabergoline treated ACTH-secreting PAs [18-20]. Only $37.1 \%$ of non-functioning (NF) PAs highly expressed D2R according to our data, consistenting with the report by Colao et al. that the cumulative evidence for NF PAs shrinkage after DA therapy is 27.6\% [21].

MGMT is a DNA repair protein that counteracts the effect of TMZ which is used for malignant glioma standard treatment. Recently, more and more studies revealed the therapeutic effect of TMZ on PAs, especially on aggressive PAs and pituitary carcinomas. MGMT expression as assessed by immunohistochemistry may predict response to temozolomide therapy in patients with aggressive pituitary tumors $[7,22]$. McCormack group demonstrated that low MGMT expression and MGMT promoter methylation were found in the pituitary tumor of the patient who responded to TMZ, high MGMT expression was seen in the patient demonstrating a poor response to TMZ [23]. They reported the results that eleven out of 88 PA samples (13\%) had low MGMT expression, and that prolactinomas were more likely to have low MGMT expression compared with other pituitary tumor subtypes. Herein, in this study we detected 170 out of 197 PAs (86.3\%) existing MGMT expression lower than $50 \%(<50 \%)$ which was considered to be low MGMT expression. This data was higher than that form reported clinical studies in TMZ treated functioning PA, non-functioning PA and pituitary carcinoma with the remission rate of $75 \%, 55 \%$ and $72 \%$ respectively, which can be explained by Bush's study that not all MGMT low expression PA respond to TMZ although medical 
Table 2 Association of D2R, MGMT and VEGF expression with clinicopathological characteristics from patients with PA

\begin{tabular}{|c|c|c|c|c|c|c|c|c|c|c|}
\hline \multirow[t]{2}{*}{ Parameters } & \multirow[t]{2}{*}{ No. of patients } & \multicolumn{2}{|l|}{$\mathrm{D} 2 \mathrm{R}$} & \multirow[t]{2}{*}{$\mathbf{P}$} & \multicolumn{2}{|c|}{ MGMT } & \multirow[t]{2}{*}{$\mathbf{P}$} & \multicolumn{2}{|c|}{ VEGF } & \multirow[t]{2}{*}{$\mathbf{P}$} \\
\hline & & Low & High & & Low & High & & Low & High & \\
\hline Cases & 197 & 69 & 128 & & 170 & 27 & & 81 & 116 & \\
\hline Gender & & & & 0.736 & & & 0.826 & & & 0.646 \\
\hline Male & 106 & 36 & 70 & & 92 & 14 & & 42 & 64 & \\
\hline Female & 91 & 33 & 58 & & 78 & 13 & & 39 & 52 & \\
\hline Aggressive & & & & 0.410 & & & 0.220 & & & 0.602 \\
\hline Yes & 64 & 25 & 39 & & 58 & 6 & & 28 & 36 & \\
\hline No & 133 & 44 & 89 & & 112 & 21 & & 53 & 80 & \\
\hline Recurrence & & & & 0.741 & & & 0.096 & & & 0.199 \\
\hline Yes & 16 & 5 & 11 & & 16 & 0 & & 9 & 7 & \\
\hline No & 181 & 64 & 117 & & 154 & 27 & & 72 & 109 & \\
\hline Tumor size & & & & 0.829 & & & 0.884 & & & 0.823 \\
\hline$\leq 10 \mathrm{~mm}$ & 16 & 6 & 10 & & 14 & 2 & & 7 & 9 & \\
\hline$>10 \mathrm{~mm}$ & 181 & 63 & 118 & & 156 & 25 & & 74 & 107 & \\
\hline Tumor texture & & & & 0.309 & & & 0.913 & & & 0.090 \\
\hline Tender & 159 & 53 & 106 & & 137 & 22 & & 70 & 89 & \\
\hline Tenacious & 38 & 16 & 22 & & 33 & 5 & & 11 & 27 & \\
\hline Bromocriptine & & & & 0.096 & & & 0.919 & & & 0.344 \\
\hline Yes & 8 & 5 & 3 & & 7 & 1 & & 2 & 6 & \\
\hline No & 189 & 64 & 125 & & 163 & 26 & & 79 & 110 & \\
\hline
\end{tabular}

Low, low expression (score of $\leq 3$ ); High, high expression (score of $>3$ ).

therapy with TMZ can be helpful in the management of life-threatening PAs that have failed to respond to conventional treatments [24]. Our results showed low MGMT expression $(<50 \%)$ in $85.7 \%$ of PRL-secreting PAs, $90 \%$ of GH-secreting PAs, $81.5 \%$ of ACTH-secreting PAs, 93.3\% of TSH-secreting PAs, $70.3 \%$ of FSH-secreting PAs and 94.3\% of non-functioning PAs, predicting almost all subtypes of PAs are suitable for TMZ therapy, although only fewer curative cases were separately reported $[25,26]$. Further large scale clinical trials are necessary.

VEGF is a key mediator of endothelial cell proliferation, angiogenesis and vascular permeability. It plays a pivotal role in the genesis and progression of solid tumors. Onofri et al. analyzed VEGF protein expression in 39 cases of PAs, found only 5 cases $(13 \%)$ were VEGF negative [8]. Lloyd et al. examined 148 human pituitary adenomas for VEGF protein expression by immunohistochemistry, and showed positive staining in all groups with stronger staining in GH, ACTH, TSH, and gonadotroph adenomas and in pituitary carcinomas [27]. Our study detected 190 positive VEGF expression cases in 197 PAs and $58.9 \%$ of them are in high expression level, including $60.7 \%$ of PRL-secreting PAs, 78.4\% FSHsecreting PAs, 51.9\% ACTH-secreting PAs and 57.1\% non-functioning PAs. Niveiro et al. investigated VEGF expression in 60 human pituitary adenomas, and found that low expression of VEGF was seen predominantly in prolactin cell adenomas, and high in non-functioning adenomas, which is different from our data that $60.7 \%$ of prolactin cell adenomas verses $57.1 \%$ non-functioning adenomas [11]. Moreover, VEGF was considered also involved in conventional medical therapy for PAs. Octreotide was reported to down-regulate VEGF expression to achieve antiangiogenic effects on PAs [28]. Gagliano et al. demonstrated that cabergoline reduces cell viability in non-functioning pituitary adenomas by inhibiting VEGF secretion, of which the modulation might mediate the effects of DA agonists on cell proliferation in nonfunctioning adenoma [29]. Interestingly, in present study, we did spearman's rank correlation analysis and found that D2R expression did not show a correlation with VEGF expression. Although it is prospective to treat PAs by anti-VEGF, up to now, only one case of PA has been reported to be cured by bevacizumab [6]. The mechanisms of VEGF in PA genesis and progression are still unclear. More studies are needed to investigate the effects of anti-VEGF therapy on PA patients.

To confirm the results, we also detected the expression of D2R, MGMT and VEGF by using western blot. The data supported the results of immunohistochemical staining. Two samples were selected for each PAs subtype. The positive expression of western blot indicated 
the immunohistochemical staining is available, and the thickness differences of the blot band revealed the expression level differences in separate sample.

Moreover, by spearman's rank correlation analysis, we found that MGMT expression was positively associated with D2R and VEGF expression in PAs. As far as we know, it is the first time to report the association of D2R and MGMT expression which is positive. Only one report by Moshkin et al. has ever mentioned the association of MGMT and VEGF expression in PA. They demonstrated a progressive regrowth and malignant transformation of a silent subtype 2 pituitary corticotroph adenoma, with significant VEGF and MGMT immunopositivity [30]. The association between VEGF and MGMT expression in PAs need further investigations, as well as D2R and MGMT expression.

In addition, we analyzed the association of D2R, MGMT and VEGF expression with clinical features of PAs, but no association was found. This indicated that their expression was not affected by the differences of clinical features, and that the medical therapy can be applied in any patient in need.

In conclusion, in this study we demonstrated the expression of D2R, MGMT and VEGF in 197 different histological subtypes of pituitary adenomas, and analyzed the relationships between D2R, MGMT and VEGF expression and the association of D2R, MGMT and VEGF expression with PA clinical features including patient sex, tumor growth pattern, tumor recurrence, tumor size, tumor tissue texture and bromocriptine application. Our data revealed that PRL-and GH-secreting PAs exist high expression of D2R, responding to dopamine agonists; Most PAs exist low expression of MGMT and high expression of VEGF, TMZ or bevacizumab treatment could be applied under the premise of indications.

\section{Abbreviations}

PA: Pituitary adenoma; D2R: Dopamine D2 receptors; DA: Dopamine agonist; MGMT: O6-methylguanine DNA methyltransferase; TMZ: Temozolomide; VEGF: Vascular endothelial growth factor; PRL: Prolactin; GH: Growth hormone; ACTH: Adrenocorticotropic hormone; TSH: Thyroid stimulating hormone; FSH: Follicle-stimulating hormone; NF: Non-functioning.

\section{Competing interests}

The authors declare that they have no competing of interests.

\section{Authors' contributions}

$Y W, J L$ and $C M$ designed the research; YW, JL, YH, MT, SW, WL and ZL performed the research; $W L$ and $Z L$ evaluated the pathological sections and scored the extent of staining; $J \mathrm{~L}$ and $\mathrm{YW}$ analyzed the data; JL, YW and $C M$ wrote the paper, CM revised the paper. All authors read and approved the final manuscript.

\section{Acknowledgements}

We thank the Department of Pathology of Jinling Hospital, School of Medicine Nanjing University, for technical support. This study was supported by National Natural Science Foundation of China (NO. 30801178).

\section{Author details}

'Department of Neurosurgery, Jinling Hospital, School of Medicine, Nanjing University, 305 East Zhongshan Road, Nanjing 210002, China. ²Department of Pathology, Jinling Hospital, School of Medicine, Nanjing University, 305 East Zhongshan Road, Nanjing 210002, China. ${ }^{3}$ Department of Neurosurgery, Yangzhou No.1 People's Hospital, The Second Clinical School of Yangzhou University, 368 Hanjiang Road, Yangzhou 225012, China.

Received: 11 February 2014 Accepted: 27 June 2014

Published: 16 July 2014

\section{References}

1. Bianchi A, Valentini F, luorio R, Poggi M, Baldelli R, Passeri M, Giampietro A, Tartaglione L, Chiloiro S, Appetecchia M, Gargiulo P, Fabbri A, Toscano V, Pontecorvi A, De Marinis L: Long-term treatment of somatostatin analogrefractory growth hormone-secreting pituitary tumors with pegvisomant alone or combined with long-acting somatostatin analogs: a retrospective analysis of clinical practice and outcomes. J Exp Clin Cancer Res 2013, 32:40. doi:10.1186/1756-9966-32-40.

2. Wan $\mathrm{H}$, Chihiro $\mathrm{O}$, Yuan S: MASEP gamma knife radiosurgery for secretory pituitary adenomas: experience in 347 consecutive cases. $J$ Exp Clin Cancer Res 2009, 28:36. doi:10.1186/1756-9966-28-36.

3. Mantovani A, Macrì A: Endocrine effects in the hazard assessment of drugs used in animal production. J Exp Clin Cancer Res 2002, 21:445-456.

4. Colao A, Pivonello R, Di Somma C, Savastano S, Grasso LF, Lombardi G: Medical therapy of pituitary adenomas: effects on tumor shrinkage. Rev Endocr Metab Disord 2009, 10:111-123.

5. Takeshita A, Inoshita N, Taguchi M, Okuda C, Fukuhara N, Oyama K, Ohashi K, Sano T, Takeuchi Y, Yamada S: High incidence of low O(6)-methylguanine DNA methyltransferase expression in invasive macroadenomas of Cushing's disease. Eur J Endocrinol 2009, 161:553-559.

6. Ortiz LD, Syro LV, Scheithauer BW, Ersen A, Uribe H, Fadul CE, Rotondo F, Horvath E, Kovacs K: Anti-VEGF therapy in pituitary carcinoma. Pituitary 2012, 15:445-449.

7. Fadul CE, Kominsky AL, Meyer LP, Kingman LS, Kinlaw WB, Rhodes $\mathrm{CH}$, Eskey CJ, Simmons NE: Long-term response of pituitary carcinoma to temozolomide. Report of two cases. J Neurosurg 2006, 105:621-626.

8. Onofri C, Theodoropoulou M, Losa M, Uhl E, Lange M, Arzt E, Stalla GK, Renner U: Localization of vascular endothelial growth factor (VEGF) receptors in normal and adenomatous pituitaries: detection of a nonendothelial function of VEGF in pituitary tumours. J Endocrinol 2006, 191:249-261.

9. Chinot OL, Barrie M, Fuentes S, Eudes N, Lancelot S, Metellus P, Muracciole X, Braguer D, Ouafik L, Martin PM, Dufour H, Figarella-Branger D: Correlation between 06-methylguanine-DNA methyltransferase and survival in inoperable newly diagnosed glioblastoma patients treated with neoadjuvant temozolomide. J Clin Oncol 2007, 25:1470-1475.

10. Dowell JE, Dunphy FR, Taub RN, Gerber DE, Ngov L, Yan J, Xie Y, Kindler HL: A multicenter phase II study of cisplatin, pemetrexed, and bevacizumab in patients with advanced malignant mesothelioma. Lung Cancer 2012, 77:567-571

11. Niveiro M, Aranda Fl, Peiro G, Alenda C, Pico A: Immunohistochemical analysis of tumor angiogenic factors in human pituitary adenomas. Hum Pathol 2005, 36:1090-1095.

12. Knosp E, Steiner E, Kitz K, Matula C: Pituitary adenomas with invasion of the cavernous sinus space: a magnetic resonance imaging classification compared with surgical findings. Neurosurgery 1993, 33:610-618.

13. Guiramand J, Montmayeur JP, Ceraline J, Bhatia M, Borrelli E: Alternative splicing of the dopamine D2 receptor directs specificity of coupling to G-proteins. J Biol Chem 1995, 270:7354-7358.

14. de Bruin C, Feelders RA, Waaijers AM, van Koetsveld PM, Sprij-Mooij DM, Lamberts SW, Hofland L: Differential regulation of human dopamine D2 and somatostatin receptor subtype expression by glucocorticoids in vitro. J Mol Endocrinol 2009, 42:47-56.

15. Colao A, di Sarno A, Pivonello R, di Somma C, Lombardi G: Dopamine receptor agonists for treating prolactinomas. Expert Opin Investig Drugs 2002, 11:787-800.

16. Verhelst J, Abs R, Maiter D, van den Bruel A, Vandeweghe M, Velkeniers B, Mockel J, Lamberigts G, Petrossians P, Coremans P, Mahler C, Stevenaert A, Verlooy J, Raftopoulos C, Beckers A: Cabergoline in the treatment of 
hyperprolactinemia: a study in 455 patients. J Clin Endocrinol Metab 1999, 84:2518-2522.

17. Sherlock M, Fernandez-Rodriguez E, Alonso AA, Reulen RC, Ayuk J, Clayton RN, Holder G, Sheppard MC, Bates A, Stewart PM: Medical therapy in patients with acromegaly: predictors of response and comparison of efficacy of dopamine agonists and somatostatin analogues. J Clin Endocrinol Metab 2009, 94:1255-1263.

18. de Bruin C, Pereira AM, Feelders RA, Romijn JA, Roelfsema F, Sprij-Mooij DM, van Aken MO, van der Lelij AJ, de Herder WW, Lamberts SW, Hofland L: Coexpression of dopamine and somatostatin receptor subtypes in corticotroph adenomas. J Clin Endocrinol Metab 2009, 94:1118-1124.

19. Pivonello R, Ferone D, de Herder WW, Faggiano A, Bodei L, de Krijger RR, Lombardi G, Colao A, Lamberts SW, Hofland LJ: Dopamine receptor expression and function in corticotroph ectopic tumors. J Clin Endocrinol Metab 2007, 92:65-69.

20. Miller JW, Crapo L: The medical treatment of Cushing's syndrome. Endocr Rev 1993, 14:443-458.

21. Colao A, Di Somma C, Pivonello R, Faggiano A, Lombardi G, Savastano S: Medical therapy for clinically non-functioning pituitary adenomas. Endocr Relat Cancer 2008, 15:905-915.

22. Syro LV, Ortiz LD, Scheithauer BW, Lloyd R, Lau Q, Gonzalez R, Uribe H, Cusimano M, Kovacs K, Horvath E: Treatment of pituitary neoplasms with temozolomide: a review. Cancer 2011, 117:454-462.

23. McCormack Al, McDonald KL, Gill AJ, Clark SJ, Burt MG, Campbell KA Braund WJ, Little NS, Cook RJ, Grossman AB, Robinson BG, Clifton-Bligh RJ: Low 06-methylguanine-DNA methyltransferase (MGMT) expression and response to temozolomide in aggressive pituitary tumours. Clin Endocrinol (Oxf) 2009, 71:226-233.

24. Bush ZM, Longtine JA, Cunningham T, Schiff D, Jane JA Jr, Vance ML, Thorner MO, Laws ER Jr, Lopes MB: Temozolomide treatment for aggressive pituitary tumors: correlation of clinical outcome with O(6)-methylguanine methyltransferase (MGMT) promoter methylation and expression. $J$ Clin Endocrinol Metab 2010, 95:E280-E290

25. Salehi F, Scheithauer BW, Moyes VJ, Drake WM, Syro LV, Manoranjan B, Sharma S, Horvath E, Kovacs K: Low immunohistochemical expression of MGMT in ACTH secreting pituitary tumors of patients with Nelson syndrome. Endocr Pathol 2010, 21:227-229.

26. Salehi F, Scheithauer BW, Kovacs K, Horvath E, Syro LV, Sharma S, Manoranjan B, Cusimano M: O-6-methylguanine-DNA methyltransferase (MGMT) immunohistochemical expression in pituitary corticotroph adenomas. Neurosurgery 2012, 70:491-496.

27. Lloyd RV, Scheithauer BW, Kuroki T, Vidal S, Kovacs K, Stefaneanu L: Vascular Endothelial Growth Factor (VEGF) expression in human pituitary adenomas and carcinomas. Endocr Pathol 1999, 10:229-235.

28. Kurosaki M, Saegert W, Abe T, Ludecke DK: Expression of vascular endothelial growth factor in growth hormone-secreting pituitary adenomas: special reference to the octreotide treatment. Neurol Res 2008, 30:518-522.

29. Gagliano T, Filieri C, Minoia M, Buratto M, Tagliati F, Ambrosio MR, Lapparelli M Zoli M, Frank G, degli Uberti E, Zatelli MC: Cabergoline reduces cell viability in non functioning pituitary adenomas by inhibiting vascular endothelial growth factor secretion. Pituitary 2013, 16:91-100.

30. Moshkin O, Syro LV, Scheithauer BW, Ortiz LD, Fadul CE, Uribe H, Gonzalez R, Cusimano M, Horvath E, Rotondo F, Kovacs K: Aggressive silent corticotroph adenoma progressing to pituitary carcinoma: the role of temozolomide therapy. Hormones (Athens) 2011, 10:162-167.

doi:10.1186/s13046-014-0056-y

Cite this article as: Wang et al:: The expression profile of Dopamine D2 receptor, MGMT and VEGF in different histological subtypes of pituitary adenomas: a study of 197 cases and indications for the medical therapy. Journal of Experimental \& Clinical Cancer Research 2014 33:56.

\section{Submit your next manuscript to BioMed Central and take full advantage of:}

- Convenient online submission

- Thorough peer review

- No space constraints or color figure charges

- Immediate publication on acceptance

- Inclusion in PubMed, CAS, Scopus and Google Scholar

- Research which is freely available for redistribution

Submit your manuscript at www.biomedcentral.com/submit
C Biomed Central 